OPEN ACCESS

Edited by:

Jiake Xu,

University of Western Australia

Australia

Reviewed by:

ZuFu Lu,

The University of Sydney, Australia

Bin Li,

Soochow University, China

*Correspondence:

Makarand V. Risbud

Makarand.risbud@jefferson.edu

Specialty section:

This article was submitted to

Molecular and Cellular Pathology,

a section of the journal

Frontiers in Cell and Developmental

Biology

Received: 27 November 2021

Accepted: 03 January 2022

Published: 03 February 2022

Citation:

Boneski PK, Madhu V, Tomlinson RE,

Shapiro $I M$, van de Wetering $K$ and Risbud MV (2022) Abcc6 Null Mice-a Model for Mineralization Disorder PXE Shows Vertebral Osteopenia Without Enhanced Intervertebral Disc Calcification With Aging. Front. Cell Dev. Biol. 10:823249. doi: $10.3389 /$ fcell.2022.823249

\section{Abcc6 Null Mice-a Model for Mineralization Disorder PXE Shows Vertebral Osteopenia Without Enhanced Intervertebral Disc Calcification With Aging}

\author{
Paige K. Boneski ${ }^{1}$, Vedavathi Madhu ${ }^{1}$, Ryan E. Tomlinson ${ }^{1}$, Irving M. Shapiro ${ }^{1}$, \\ Koen van de Wetering $^{2}$ and Makarand V. Risbud ${ }^{1 *}$
}

${ }^{1}$ Department of Orthopedic Surgery, Thomas Jefferson University, Philadelphia, PA, United States, ${ }^{2}$ Department of Dermatology and Cutaneous Biology, Jefferson Institute of Molecular Medicine and PXE International Center of Excellence in Research and Clinical Care, Sidney Kimmel Medical College, Thomas Jefferson University, Philadelphia, PA, United States

Chronic low back pain is a highly prevalent health condition intricately linked to intervertebral disc degeneration. One of the prominent features of disc degeneration that is commonly observed with aging is dystrophic calcification. ATP-binding cassette sub-family $\mathrm{C}$ member 6 (ABCC6), a presumed ATP efflux transporter, is a key regulator of systemic levels of the mineralization inhibitor pyrophosphate (PPi). Mutations in ABCC6 result in pseudoxanthoma elasticum (PXE), a progressive human metabolic disorder characterized by mineralization of the skin and elastic tissues. The implications of ABCC6 loss-of-function on pathological mineralization of structures in the spine, however, are unknown. Using the $A b c c 6^{-/-}$mouse model of PXE, we investigated age-dependent changes in the vertebral bone and intervertebral disc. $A b c c 6^{-1-}$ mice exhibited diminished trabecular bone quality parameters at 7 months, which remained significantly lower than the wild-type mice at 18 months of age. $A b c c 6^{-/-}$vertebrae showed increased TRAP staining along with decreased TNAP staining, suggesting an enhanced bone resorption as well as decreased bone formation. Surprisingly, however, loss of ABCC6 resulted only in a mild, aging disc phenotype without evidence of dystrophic mineralization. Finally, we tested the utility of oral K3Citrate to treat the vertebral phenotype since it is shown to regulate hydroxyapatite mechanical behavior. The treatment resulted in inhibition of the osteoclastic response and an early improvement in mechanical properties of the bone underscoring the promise of potassium citrate as a therapeutic agent. Our data suggest that although ectopic mineralization is tightly regulated in the disc, loss of ABCC6 compromises vertebral bone quality and dysregulates osteoblast-osteoclast coupling.

Keywords: ectopic mineralization, osteopenia, intervertebral disc degeneration, spine, vertebra bone, potassium citrate, nucleus pulposus, annulus fibrosus 


\section{INTRODUCTION}

The ABCC6 protein is a mediator of cellular ATP release (Jansen et al., 2013; Jansen et al., 2014) into the blood from hepatocytes where it is primarily expressed. ABCC6 inhibits ectopic mineralization, wherein extracellular ATP is converted to AMP and the key mineralization inhibitor PPi via ectonucleotide pyrophosphate/phosphodiesterase 1 (ENPP1). Absence of ABCC6 causes pseudoxanthoma elasticum (PXE), an autosomal recessive metabolic disorder characterized by ectopic mineralization in elastin-rich tissues such as the eyes, blood vessel walls, and the skin (Borst et al., 2019). To date, over 300 mutations in the ABCC6 gene have been identified, the majority of which are single nucleotide missense mutations that result in protein function loss (Favre et al., 2017). Consequently, global ABCC6 knockout mouse is commonly used to study PXE phenotypes (Bergen et al., 2000; Li et al., 2017). Noteworthy, while ABCC6 is highly expressed in the liver and kidneys, it is minimally expressed, or absent, in elastin-rich tissues affected by PXE (Klement et al., 2005). The PPi produced in the liver is distributed to peripheral organs through blood, with ABCC6 contributing about $60-70 \%$ of plasma PPi (Jansen et al., 2013; Jansen et al., 2014) underscoring the metabolic nature of PXE.

Mineral homeostasis is under tight regulation to maintain appropriate development of various tissues including cartilage, bone, and other connective tissues. When mineralization is dysregulated, however, it can result in ectopic mineralization, the aberrant deposition of calcium-phosphate hydroxyapatite crystals in soft connective tissues (Li et al., 2014). Ectopic mineralization affects the connective tissues of the spinal motion segment including the intervertebral disc, facet joint cartilage, and ligaments (Hristova et al., 2011). While disc degeneration is multifactorial, one of the disease subphenotypes is ectopic calcification of proteoglycan rich nucleus pulposus (NP) and cartilaginous endplates (CEP) (Moskowitz et al., 1990; Roberts et al., 1998), where the latter has been proposed to cause changes to the blood supply and block diffusion of nutrients into the disc (Ubran et al., 1976; Clarke et al., 2008). In a recent study using high-resolution imaging digital-contact radiography, a strong correlation between aging, disc degeneration status, and pathological calcification of intervertebral disc and between disc and facet joint degeneration and facet cartilage calcification has been shown (Hawellek et al., 2017). Noteworthy, 100\% disc and 36.5\% facet cartilage specimens showed calcifications indicating that pathological calcification is a prominent feature of the degenerative process (Hawellek et al., 2017). Several mouse models such as spontaneous Enpplasj-2J mutant mice (Zhang et al., 2016), a model of generalized arterial calcification of infancy (GACI) and aging inbred LG/J mice (Novais et al., 2020) have shown high incidence of disc calcification linked to degeneration that affects the fibrocartilaginous annulus fibrosus (AF) and NP compartments (Novais et al., 2020). Other ectopic mineralization disorders such as diffuse idiopathic skeletal hyperostosis (DISH), caused by a lack of equilibrative nucleoside transporter 1 (ENT1), is characterized by increased ectopic mineralization of AF tissue and bone mineral density which progresses with age (Warraich et al., 2013; Fournier et al., 2020). These ENT1 null mice show reduced expression of anti-mineralization genes $M g P, E n p p 1$, and Spp 1 by 6 months of age and enhanced ectopic spine calcification from cervical to caudal regions in an age-dependent manner (Ii et al., 2016). Surprisingly, reports of PXE-related calcifications in the musculoskeletal system have only until recently been investigated in zebrafish, where the knockout of the ABCC6 ortholog resulted in hypermineralization of the axial skeleton (Mackay et al., 2015). A very recent study showed that a small subset, 16.7\%, of adult GACI patients with mutations in ABCC6 suffered from hypophosphatemic rickets (Ferreira et al., 2021), implying that bone is an affected tissue in relation to ABCC6 mutations. It is also important to note that the intervertebral disc contains an extensive elastin fiber network (Yu et al., 2007), which increases with aging and degeneration (Cloyd and Elliott, 1976). Consequently, discs could be susceptible to mineralization in the context of PXE spectrum of disorders. Given that PPi is a major inhibitor of connective tissue mineralization, it is unclear whether ABCC6-derived systemic PPi is important for preventing disc calcification.

Here, for the first time we provide insights into the role of ABCC6 in vertebrae and the intervertebral disc in the context of aging using Abcc6 knockout mice. Our results show that loss of ABCC6 significantly affects the vertebral bone morphology and mass. Moreover, we show that K3Citrate could have a potential in treating the observed osteopenic phenotype due to its known propensity to accumulate in bones where it plays an important function in regulating hydroxyapatite mechanical behavior ( $\mathrm{Hu}$ et al., 2010). Interestingly, we observed that ABCC6 loss does not predispose discs to pathological mineralization. These results highlight the significance of local vs. systemic regulation of $\mathrm{PPi}$ metabolism in the musculoskeletal system.

\section{MATERIALS AND METHODS}

\section{Mice}

All animal experiments were conducted following Institutional Animal Care and Use Committee (IACUC) protocols approved by Thomas Jefferson University. In total, 23-weeks, 7 to 8months, 12-months, and 16- to 18-month-old C57BL6J mice with global deletion of ABCC6, a model for PXE, were analyzed (Klement et al., 2005). After weaning, at 3 weeks of age, mice treated with potassium citrate (K3Citrate) received either $2 \mathrm{mM}$ or $40 \mathrm{mM}$ orally through drinking water for 20 weeks and sacrificed at 23 weeks old.

\section{Micro-CT Analysis}

Micro-CT scans (Bruker SkyScan 1275) of fixed spines were conducted under the following parameters: $50 \mathrm{kV}$ voltage, $200 \mu \mathrm{A}$ current, $15 \mu \mathrm{m}$ voxel size resolution. Image reconstruction was performed using NRecon Reconstruction software. Vertebral length, disc height, and DHI were calculated as previously reported (Tsingas et al., 2020). CTan software was used for 3D data analysis of the trabecular bone and 
to assess bone volume fraction (BV/TV), trabecular thickness (Tb.Th.), trabecular number (Tb.N.), trabecular spacing (Tb.Sp.), structural model index (SMI), and bone mineral density (BMD). $2 \mathrm{D}$ analysis of cortical bone assessed bone area (B.Ar.), crosssectional thickness (Cs.Th.), mean polar moment of inertia (MMI), and tissue mineral density (TMD).

\section{Histological Analysis}

Spines were dissected and fixed in 4\% PFA for $48 \mathrm{~h}$, decalcified in 20\% EDTA for 15 days, and lumbar (L3-L6) and caudal (Ca1Ca6) discs were embedded in paraffin. Samples used for calcified sections (L1-L3 and Ca6-Ca12) were placed in 30\% sucrose solution for $36 \mathrm{~h}$ immediately following fixation, then embedded in OCT and snap frozen. There were $7 \mu \mathrm{m}$ thick mid-coronal sections stained with 1\% Safranin-O, 0.05\% Fast Green, and $1 \%$ Hematoxylin and Picrosirius Red for morphological assessment and collagen localization, respectively. There were $10 \mu \mathrm{m}$ thick sections prepared for calcified samples that were collected as previously reported (Dyment et al., 2016) and stained with Alizarin Red to detect free calcium and mineralized structures. Slides were imaged using Axio Imager 2 microscope (Carl Zeiss) equipped with Axiocam 105 color camera with a $5 \mathrm{x} / 0.15 \mathrm{~N}$-Achroplan objective and Zen2 ${ }^{\text {TM }}$ software (Carl Zeiss), and Eclipse LV100 POL polarizing microscope with a $5 x / 0.15$ objective (Nikon) and NIS Elements Viewer software. Gross histology was analyzed using a modified Thompson grading scale and the scores of $\mathrm{n} \geq 4$ blind graders. Endplate disorganization was analyzed using an endplate grading scale as previously reported (Tessier et al., 2020); scoring was performed by $n \geq 3$ blind graders. Grading was performed for three lumbar and five caudal discs per mouse [23W ( $n=8 \mathrm{WT}, 8 \mathrm{KO}), 128$ discs total; $7-8 \mathrm{M}(n=7 \mathrm{WT}$, five $\mathrm{KO}$ ), 96 discs total; $12 \mathrm{M}$ ( $n=5 \mathrm{WT}, 6 \mathrm{KO}$ ), 80 discs total; $16-18 \mathrm{M}$ $(n=8 \mathrm{WT}, 8 \mathrm{KO}), 128$ discs total].

\section{Immunohistology}

Mid-coronal sections were deparaffinized and rehydrated using an ethanol series. Following appropriate antigen retrieval, sections were blocked in the appropriate blocking solution (5-10\% normal goat serum or normal donkey serum) and then incubated with primary antibodies against carbonic anhydrase 3 (1:150, SantaCruz), keratin 19 (1:50, DSHB, TROMA-III), COMP (1:200, Abcam, ab231977), COL I (1:100, Abcam, ab34710), COL II (1:400, Fitzgerald Industries, R-CR008), COL X (1:500, Abcam, ab58632), aggrecan (1:50, Millipore, AB1031) in a blocking buffer at $4^{\circ} \mathrm{C}$ overnight. ARGxx (1:200, Abcam, ab3773) and CS (Abcam ab11570) used a MOM kit (Vector Laboratories, Burlingame, CA, United States) for staining and incubation of primary antibodies. Sections were washed with PBS and incubated with conjugated secondary antibody Alexa-Fluor-594 (Jackson ImmunoResearch Lab, Inc., 1:700) for $1 \mathrm{~h}$ at room temperature. Sections were stained to detect activity of tartrate-resistant acid phosphatase (1:80, Invitrogen, E6601A) and alkaline phosphatase (Vector Laboratories, SK-5300). All sections were mounted with DAPI (Fisher Scientific, P36934) and imaged using Axio Imager 2 microscope equipped with AxioCam
MRm monochrome camera with a $5 \mathrm{x} / 0.15 \mathrm{~N}$-Achroplan or $20 \mathrm{x} /$ 0.5 EC Plan-Neofluar objectives and Zen2TM software (Carl Zeiss). For quantification, percent-stained area, cell count, and cell band area were calculated by thresholding, transforming the image to binary, and measuring particle number and area using ImageJ software, v1.53a, last access 9/11/2021 https://imagej.nih. gov/ij/.

\section{Tissue RNA Isolation and Microarray Analysis}

AF tissue from lumbar discs (L1-L6) of 7-month-old wild-type ( $n=4$ female) and $A b c c \sigma^{1-}$ mice $(n=4$ female) were collected and pooled for each individual mouse. RNA was isolated using RNeasy Mini Kit (Qiagen). Purified RNA was used to prepare cDNA using GeneChip WT Plus kit (Thermo Fisher) and hybridized using Mouse Clarion S gene chips. Gene chips were scanned using an Affymetrix Gene Chip Scanner 3000 7G and Command Console Software. Experimental quality control, analyses, and visualizations were performed using Transcriptome Analysis Console v4.0.2 (TAC). Abcc6 ${ }^{-1-}$ samples were compared to WT samples and included probe sets where at least $50 \%$ of the samples had a DABG (detected above background) of $p \leq 0.05$. Inclusion cutoffs were defined at a 1.75 -fold change and $p$-value $\leq 0.01$. Enrichment analysis of annotated genes was performed using PANTHER overrepresentation test, GO biological and molecular database annotations, and binomial statistical test with FDR $\leq$ 0.05. Array data are deposited in the GEO database (GSE188943).

\section{Biomechanical Analysis}

Vertebrae (L1-L2) from 23-week-old mice ( $n=10 \mathrm{WT}, 9 \mathrm{KO}, 4$ $\mathrm{KO} 2 \mathrm{mM}, 8 \mathrm{KO} 40 \mathrm{mM}$ ) were isolated and stored in PBS-soaked gauze at $-20^{\circ} \mathrm{C}$ before use. Mechanical loading was conducted using a material testing system (TA Systems Electroforce 3200 Series II). First, each vertebra was individually potted into a $2-\mathrm{mm}$ plastic ring mold using acrylic resin (Ortho-Jet, Patterson Dental, Saint Paul, MN). Next, a 0.4-N compressive preload was applied, followed by a monotonic displacement ramp at $0.1 \mathrm{~mm} / \mathrm{s}$ until failure. Force-displacement data were digitally captured at $25 \mathrm{~Hz}$ and converted to stress-strain using a custom GNU Octave script with micro-CT based geometric measurements, as previously described (Park et al., 2019).

\section{Statistical Analysis}

All statistical analyses were conducted using Prism 8 (GraphPad). Graphical data are represented as mean \pm SD, distribution was checked with a Shapiro-Wilk test for normality, and differences between the two groups were assessed by $t$-test or Mann-Whitney test when appropriate. Differences between the distribution of percentages were assessed using a Chi-squared test, and the differences between more than two groups were assessed by ANOVA or Kruskal-Wallis followed by uncorrected Dunn's multiple comparison test for non-normally distributed data. Statistical significance was considered $p \leq 0.05$. 

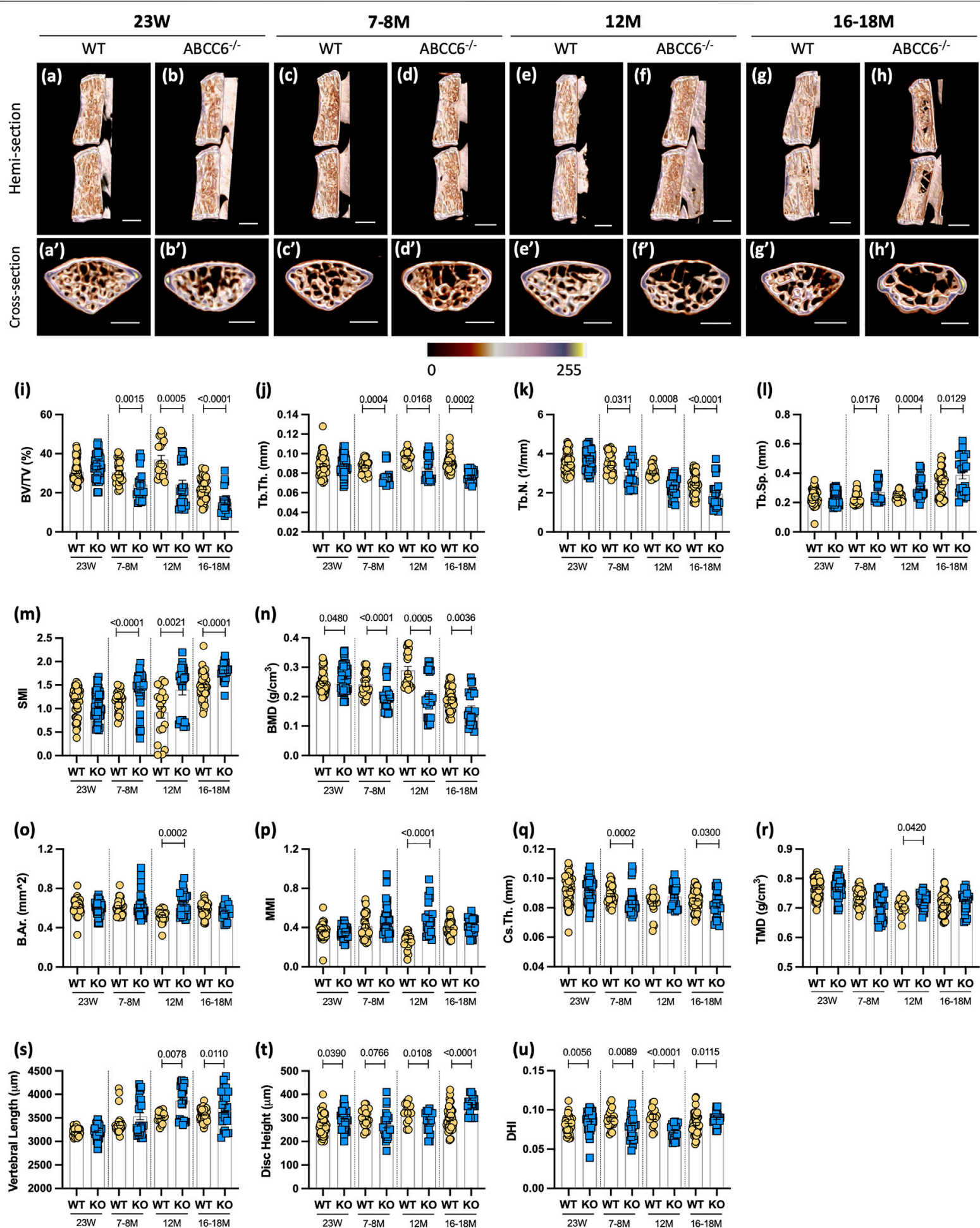

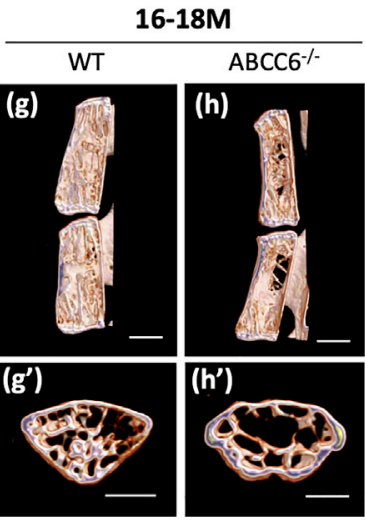

(k)
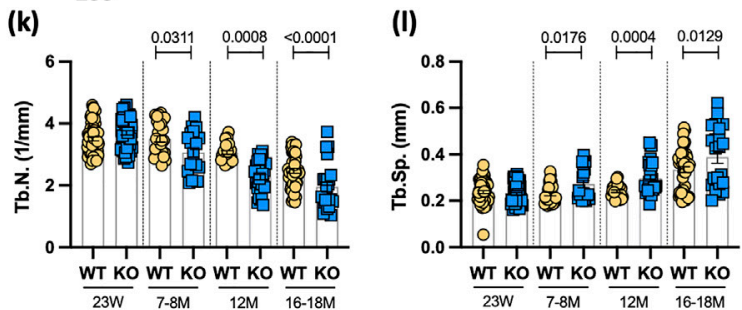

FIGURE $1 \mid \mathrm{Abcc6}^{-/-}$mice show vertebral osteopenia, altered disc height, and vertebral length. Representative microCT reconstructions of (A-H) hemi- and $\left(\mathbf{A}^{\prime}-\mathbf{H}^{\prime}\right)$ cross-sections show consistent trabecular thinning in lumbar vertebrae of $A b c c 6^{-/-}$mice at all ages. Quantitative micro-CT analysis of trabecular bone parameters (I-N) BV/TV, Tb.Th., Tb.N., Tb.Sp., SMI, BMD, and cortical bone parameters (O-R) B.Ar., MMi, Cs. Th., and TMD. (S) Vertebral length, (T) disc height, and (U) $\mathrm{DHI}$ are shown for lumbar motion segments. Quantitative analyses are shown as mean $\pm \mathrm{SD}$ ( $n=3$ lumbar discs and $n=4$ vertebrae/mouse, $n \geq 5$ mice/

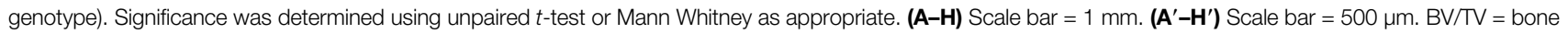
volume/tissue volume. Tb.Th. = trabecular thickness. Tb.N. = trabecular number. Tb.Sp. = trabecular spacing. SMl = structural model index. BMD = bone mineral density. B.Ar. = bone area. $\mathrm{MMI}=$ mean polar moment of inertia. Cs. Th. = cross-sectional thickness. DHI = disc height index. 


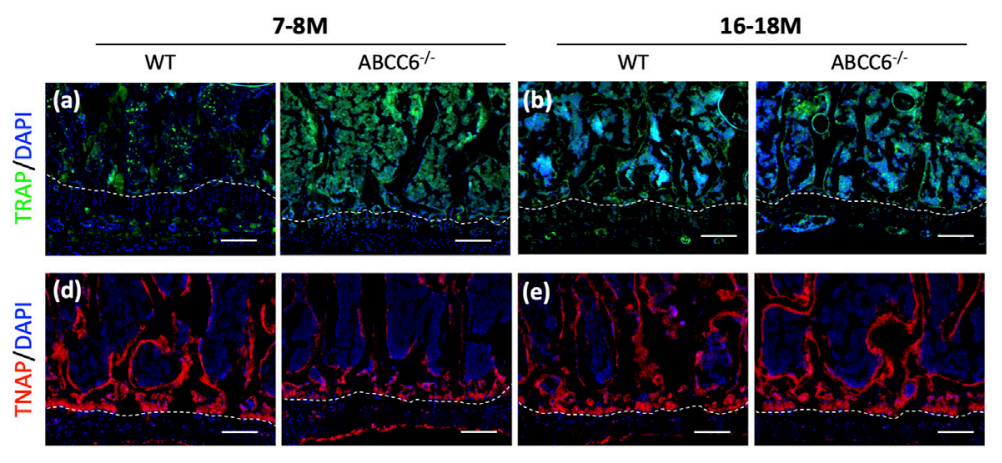

(c)

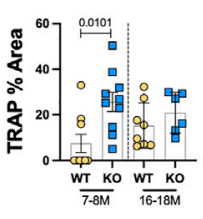

(f)

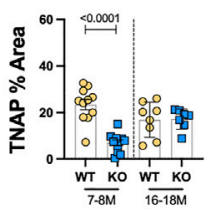

FIGURE 2 | Osteoblastic activity is dysregulated in lumbar vertebrae of $A b c c 6^{-/-}$mice. Immunohistological (IHC) staining showed increased (A,B) TRAP staining and decreased (D,E) TNAP staining in 7-8-months $A b c c 6^{-/-}$lumbar vertebrae. No difference between genotypes was observed in TRAP (C) and TNAP (F) activity in 16-18-months mice. Scale bar $=100 \mu \mathrm{m}$. Quantitative analyses are shown as mean \pm SD. ( $n=2$ vertebrae/mice, $n \geq 5$ mice/genotype/stain). Significance was determined using unpaired $t$-test or Mann Whitney test as appropriate.

\section{RESULTS}

\section{The Abcc6 $^{-/-}$Mice Show Vertebral Osteopenia, Alterations in Disc Height, and Reduced Osteoblast and Osteoclast Activity}

Changes in vertebral bone properties of PXE patients or animal models have not been previously reported. Micro-CT analysis of $A b c c \sigma^{--}$vertebrae, however, showed robust age-dependent decline in trabecular bone attributes in lumbar (Figure 1) and caudal (Supplementary Figure S1) spine and alterations in the disc height (Figures $\mathbf{1 A}-\mathbf{H}^{\prime}$ ). Trabecular parameters BV/TV (Figure 1I), Tb.Th. (Figure 1J), and Tb.N. (Figure 1K) were reduced in $A b c c \sigma^{/-}$mice, with a concomitant increase in Tb.Sp. (Figure 1L) at all timepoints except at 23 weeks. Structural model index (SMI), a parameter that determines the rod-like structure of trabeculae and is correlated to bone strength and fracture risk, was significantly higher at all timepoints except 23 weeks (Figure $1 \mathbf{M}$ ), suggesting that $A b c c \sigma^{\prime-}$ trabeculae have decreased bone strength and higher fracture risk. This observation was also supported by an initial higher bone mineral density (BMD) at 23 weeks, but then a progressive reduction in BMD from 7-8 months onward (Figure 1N). Furthermore, these changes in lumbar trabecular bone in $A b c c 6^{-1}$ mice were confirmed in the caudal spine (Supplementary Figures S1I-N). Micro-CT analysis showed less of an impact of absence of ABCC6 on lumbar cortical bone. Only at 12 months was there an increase in bone area (B.Ar.) (Figure 10), mean polar moment of inertia (MMI) (Figure 1P), and tissue mineral density (TMD) (Figure 1R). Interestingly, cross-sectional thickness (Cs.Th.) was only significantly reduced at 7-8 months and 16-18 months, whereas it trended higher at 12 months (Figure 1Q). Cortical bone in caudal vertebrae of $A b c c 6^{-/-}$was relatively unaffected with a slightly decreased MMI at 12 months and decreased TMD at 23 weeks (Supplementary Figures S1O-R). When assessing the relationship between the disc compartment and vertebrae, $A b c c 6^{-1-}$ mice had increased vertebral length and alterations in disc height and disc height index (DHI) (Figures 1S-U), parameters correlated with disc degeneration. Caudal vertebral length, disc height, and DHI, however, only showed significant differences at 7-8 months (Supplementary Figures S1S-U). Together, these results clearly showed that loss of ABCC6 altered bone mass and morphology and resulted in an ageprogressive osteopenic phenotype.

Interestingly, $A b c c \sigma^{-1-}$ vertebrae showed a significant increase in TRAP staining, associated with the osteoclast activity (Figures 2A-C), and a concomitant decrease in TNAP levels (Figures 2D-F) at 7-8 months of age. This initial dysregulation in osteoblastic activity is in line with the observed morphometric deficits suggesting that upregulated osteoclastic activity is driving this progressive trabecular bone loss. These changes, however, were not maintained at 16-18 months with TRAP expression showing only a trend of increased activity over agematched wild-type control mice, implying that over time the loss of bone is maintained due to decoupling of bone formation and resorption.

\section{Loss of ABCC6 Causes Small Changes in Cellular Phenotype but Does Not Promote Age-Dependent Disc Degeneration or Mineralization}

Although there are numerous reports on ectopic mineralization of elastin-rich tissues resulting from ABCC6 loss-of-function, there have been none that investigate the significance of this loss on the intervertebral disc. Histological analysis did not show conspicuous changes in lumbar NP and AF cell morphology (Figures 3A-B'). A modified Thompson grading scheme of NP and $\mathrm{AF}$ tissue revealed age-related disc degeneration in both wildtype and $A b c c \sigma^{-1}$ mice, particularly in the $\mathrm{AF}$, a higher proportion of discs scored a grade 3 in the $\mathrm{AF}$ at 16-18 months compared to WT discs (Figure 3C). On the other hand, $\mathrm{Abcc}^{-1}$ at $16-18$ months showed fewer discs with grade $2 \mathrm{NP}$ (Figure 3D). We also performed endplate grading using a 3-point scheme by Tessier and colleagues (Tessier et al., 2020). Interestingly, the distribution of endplate 


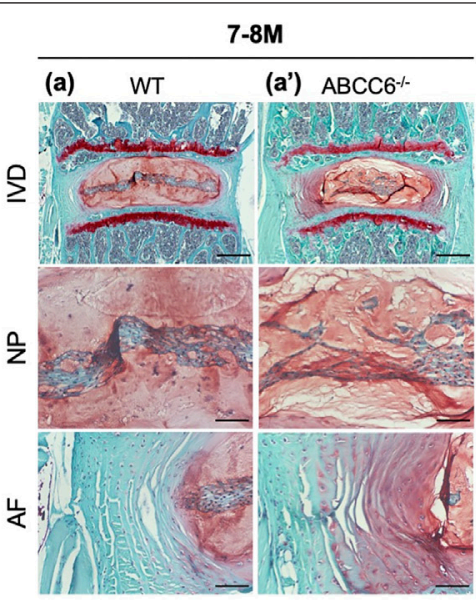

7-8M
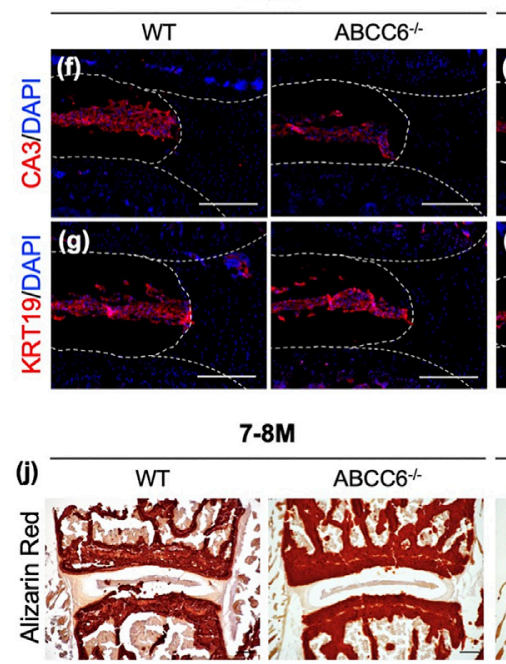

16-18M

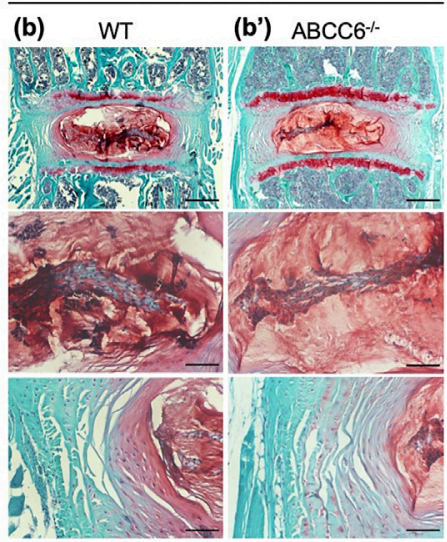

16-18M

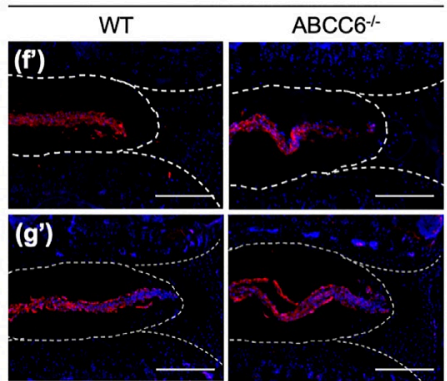

6-18M

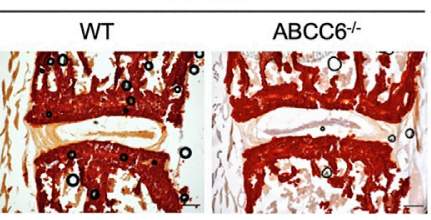

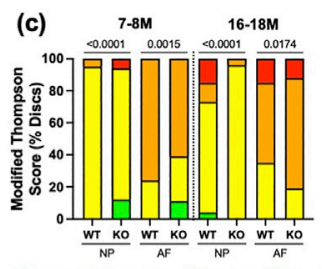
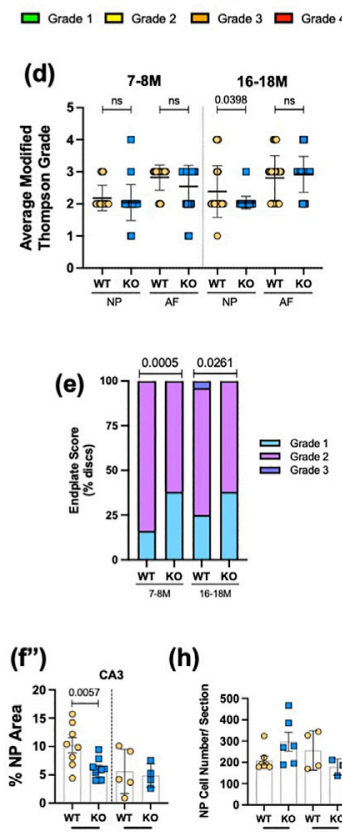

(h)

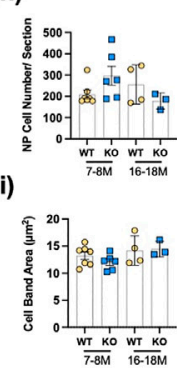

FIGURE 3|ABCC6 loss shows mild degenerative changes but does not promote disc mineralization. (A-B') Safranin O/Fast Green staining of (A-A') 7-8 months and (B-B') 16- to 18-month-old lumbar discs showed tissue morphology and proteoglycan content consistent with age-related disc degeneration (row 1, scale bar = $200 \mu \mathrm{m}$ and rows 2-3, scale bar $=50 \mu \mathrm{m})$. (C,D) Histological grading analysis using the modified Thompson scale showed changes in distribution of grades of degeneration of NP and AF but comparable average grades of degeneration. (E) Distribution of endplate grades of 7-8M and 16- to 18-month-old lumbar discs show lower endplate scores in Abcc6 ${ }^{-/-}$discs. (F-G'") $1 \mathrm{HC}$ staining of NP phenotypic markers of 7- to 8- and 16= to 18-month-old lumbar discs showed a slight decrease in (F-F") CA3 expression but no difference in (G-G") KRT19 expression. (H) Average NP cell count and (I) average NP cell band area showed no difference between genotypes. (J) Representative Alizarin red staining of 7- to 8- and 16- to 18-month-old lumbar discs showed no changes in free calcium staining within the NP or AF. Scale bar $=200 \mu \mathrm{m}$. Dotted lines denote NP and AF tissue compartments. Quantitative analyses are shown as mean \pm SD. Significance of grading distribution was determined using a $\chi^{2}$ test. Significance of average grade data and percent area were determined using unpaired $t$-test or Mann Whitney test as appropriate.

grades showed lower scores in 7-8 months and 16- to 18-monthold $\mathrm{Abcc6}^{-1-}$ mice (Figure 3E).

To investigate the phenotype of NP cells of $A b c c 6^{-1-}$ mice, levels of phenotypic markers carbonic anhydrase 3 (CA3) and keratin-19 (KRT19) were measured. There was a reduction in CA3 abundance at 7-8 months (Figures 3F-F") with a similar trend in KRT19 levels (Figures 3G-G"). However, this early reduction in marker abundance was not sustained as discs aged, suggesting that loss of ABCC6 does not exacerbate age-related decline in cell number and their phenotype (Figures 3H,I). In addition, Alizarin red staining was performed to assess calcium levels. Analysis revealed presence of AF calcification in one 16-18M $A b c c \sigma^{--}$mouse (Supplementary Figure S2A), however, there was no overall apparent elevation in calcium staining or the presence of calcified nodules in $A b c c 6^{-1-}$ discs (Figure 3J), suggesting that discs are not susceptible to pathological mineralization following the loss of ABCC6.

\section{Loss of ABCC6 Causes Alteration in Collagen Fiber Thickness and Composition in AF}

We performed Picrosirius red staining followed by polarized imaging of WT and KO lumbar discs to investigate changes in AF collagen fiber thickness (Figures $\mathbf{4} \mathbf{A}-\mathbf{B}^{\prime}$ ). The analysis of polarized images showed that lumbar discs of $\mathrm{Abcc}^{1-}$ mice 


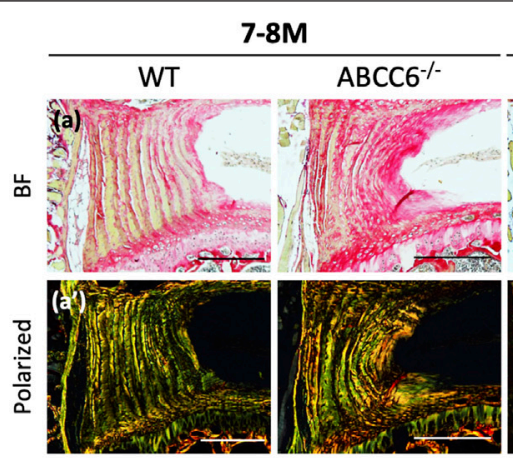

$-8 M$
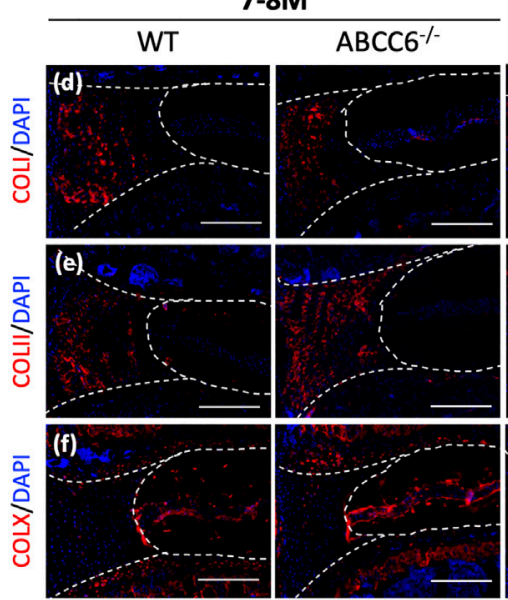

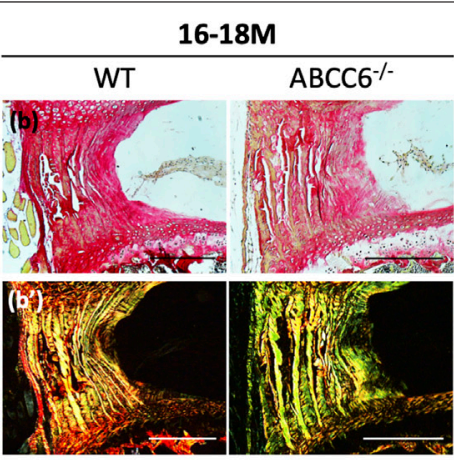

(c)

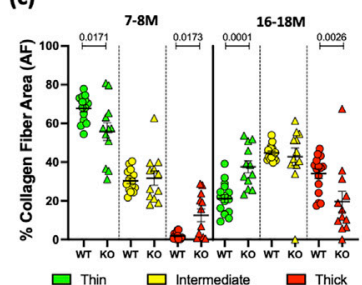

FIGURE $4 \mid \mathrm{Abcc6}^{-1-}$ mice show altered AF collagen fiber composition. (A-B') Picrosirius red staining showed altered collagen fiber thickness in the AF of $A b c c 6^{-/-}$ lumbar discs under bright field (BF) and polarized light. (C) Quantification of percent collagen fiber area showed a significant difference in distribution of fiber maturity. (D-F"') IHC staining showed no differences in (D-D") COLI and (E-E") COLIl abundance but increased (F-F"') COLX expression at both timepoints of ABCC6 ${ }^{-/-}$AF tissue. Dotted lines denote NP and AF tissue compartments. Scale bar $=200 \mu \mathrm{M}$. ( $n=1-2$ discs/animal; $n \geq 3$ mice/genotype, $5-14$ discs/genotype/stain). Quantitative analyses are shown as mean \pm SD. Significance was determined using unpaired $t$-test or Mann Whitney test as appropriate.

showed an increased proportion of thin collagen fibers and a lower proportion of thick fibers at 7-8 months of age compared to WT discs (Figures $\mathbf{4 A}^{\prime}, \mathbf{C}$ ). Interestingly, these percentages were reversed at 16-18 months with more thin fibers compared to thick fibers, suggesting that loss of ABCC6 causes a disruption in collagen turnover and homeostasis. A similar trend in collagen fiber thickness was observed in caudal discs (Supplementary Figures S2E-G). To gain further insights into compositional changes in collagen subtypes, levels of collagen-I (COLI), collagen-II (COLII), and collagen-X (COLX) were measured. Interestingly, abundance of COLI (Figures 4D-D") and COLII (Figures 4E-E") in the AF compartment was comparable between genotypes, suggesting that the changes in proportion of fiber types were not due to COLI and COLII and that other fibrillar collagens could be involved. Notably, levels of COLX, a marker of hypertrophic chondrocytes in the growth plate, were significantly elevated in both the NP and AF of 16-18 months and higher in the NP of 7-8-months $\mathrm{ABCC}^{-/-}$discs (Figures 4F-F'"). Overall, these results show that ABCC6 loss results in changes in collagen configuration that may reflect mild disc degeneration.

\section{Abcc6 $^{-/-}$Intervertebral Discs Show Minor Compositional Changes in Non-Collagenous Matrix Components}

The impact of $A b c c 6$ deletion on composition of major, noncollagenous ECM components of disc was assessed. Staining for ARGxx, an ACAN neoepitope generated by ADAMTSdependent degradation, showed a small trend of increase in the $\mathrm{AF}$ compartment of $\mathrm{ABCC6}^{-1-}$ mice at 7-8 months, whereas a significant decrease was noted at $16-18$ months (Figures 5A-A"). Aggrecan (ACAN), a high molecular weight and keratan sulfate substituted proteoglycan, was highly expressed in the NP compartment and showed increased abundance in 16- to 18-month-old $A b c c 6^{-1-}$ mice; comparable levels in NP were noted between genotypes at 7-8 months (Figures 5B-B'"'). There were no differences in aggrecan levels in the AF compartment between $\mathrm{KO}$ and WT mice at both time points. While no changes were seen in the NP compartment, levels of CS were reduced in the AF of 16- to 18-months-old $A b c c 6^{-1-}$ discs with a trend of decreasing levels at 7-8 months (Figures 5C-C'"'). Furthermore, cartilage-oligomeric matrix protein (COMP), another important non-collagenous matrix 


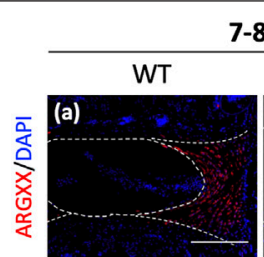

7-8M
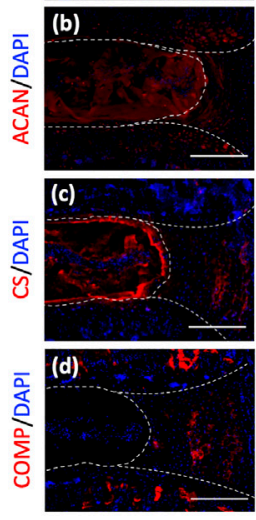

ABCC6 $\%$
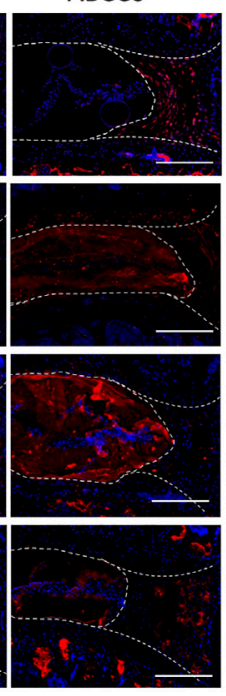

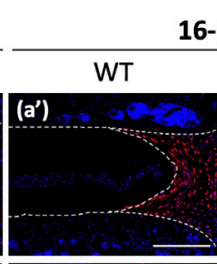

16-18M
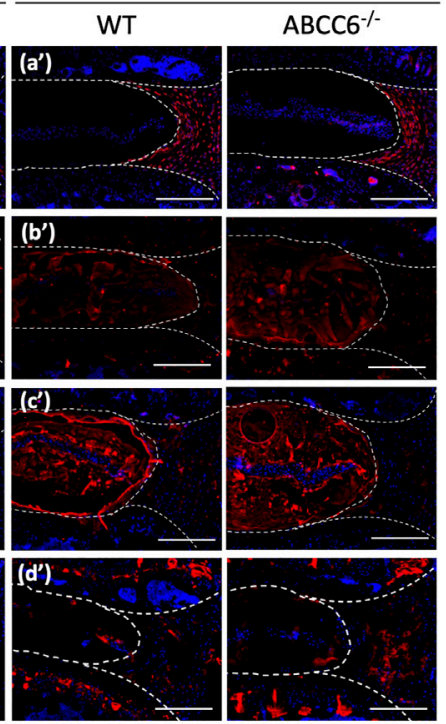

(b")
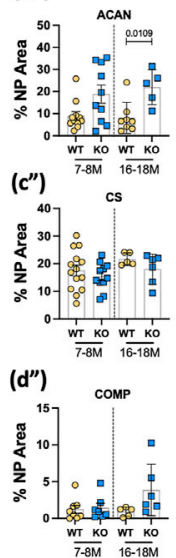

(a)

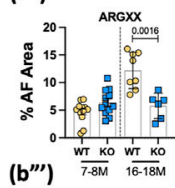

(b')
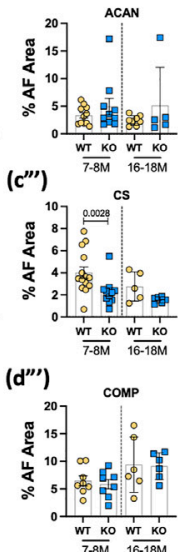

FIGURE 5 | $\mathrm{Abcc6}^{-/-}$mice exhibit changes in non-collagenous disc matrix composition. (A-D') IHC staining showed reduced expression of (A-A"') ARGXX and (C-C'") CS in the AF, increased expression of (B-B"') ACAN in the NP, and no changes in (D-D"') COMP expression in 16-18 months lumbar discs. Scale bar = $200 \mu M$. ( $n=1-2$ discs/animal; $n \geq 3$ mice/genotype, 5-14 discs/genotype/stain). Quantitative analyses are shown as mean \pm SD. Significance was determined using unpaired t-test or Mann Whitney test as appropriate.

component, showed comparable abundance across ages and genotypes (Figures 5D-D ${ }^{\prime \prime \prime}$ ). Taken together, these results suggest alteration in turnover and CS-substitution of ACAN in the AF with concomitant compensatory increase in ACAN levels in the NP of $A b c c \sigma^{-1-}$ mice.

\section{Abcc6 $^{-/-}$Mice Reveal Transcriptomic Changes Related to Metabolic Processes and Sensory Perception}

Since AF tissue has an extensive elastin network and undergoes ectopic mineralization in humans and mouse models of disc degeneration, we performed microarray analysis of AF tissue from 7-month-old mice with an aim to obtain molecular and mechanistic insights into early transcriptomic changes resulting from ABCC6 loss. This age also corresponded with emergence of an osteopenic phenotype in vertebrae. Three-dimensional principal component analysis (PCA) showed clustering of WT samples and consistent clustering among three of the four $\mathrm{KO}$ samples (Figure 6A). However, when a stringent cutoff of FDR $\leq$ 0.5 with a fold change of $\geq 1.75$ was used, it did not find any DEGs between the genotypes. Consequently, to ensure that we capture all the relevant transcriptomic changes resulting from the loss of $A b c c 6$, we used a cutoff of $p \leq 0.01$ with a fold change of $\geq 1.75$ resulting in a list of $144 \mathrm{DEGs}$ in $A b c c 6^{-1-} \mathrm{AF}$ tissue (Figure 6B). The hierarchical clustering heatmap and volcano plot were used to visualize the directionality and proportions of DEGs (Figures 6C,D).

Enrichment analysis was performed using PANTHER to assess GO biological, molecular, and cellular pathways related to these DEGs. From this analysis, among the most enriched upregulated GO biological and cellular processes included sensory perception, cellular metabolic process, signal transduction, $G$ protein-coupled receptor activity, and ion binding (Figures 6E,F). Among the most enriched downregulated GO processes included cellular macromolecule molecular process, protein metabolic process, and cellular processes related to clathrin coat and membrane-bound organelle (Figure 6G). The select genes classified under these enriched pathways included upregulation of signal transduction genes Ifna12, Npy6r, Bcl2a1d, and Ptgfr and metabolic process genes Klk1b22 and Egln3. Whereas downregulated DEGs included Mmp12, Hdac2, and Tspan5 (Figures 6I,J). Overall, the clustering of these DEGs in more generalized biological processes and cellular pathways is consistent with the observed intervertebral disc phenotype of $A b c c \sigma^{-/}$mice which lack ectopic mineralization and pronounced degeneration.

\section{K3Citrate Treatment Restores Early Cellular Changes and Mechanical Performance in Abcc6 $^{-/-}$Vertebrae}

We investigated whether oral supplementation K3Citrate can reverse the early cellular and mechanical changes that may occur prior to the onset of bone loss. We analyzed vertebrae from 23week-old $A b c c \sigma^{-1-}$ mice that were treated with vehicle or K3Citrate at 2 and $40 \mathrm{mM}$ for 20 weeks starting at 3 weeks of age. While 23-week-old $A b c c \sigma^{1-}$ mice do not evidence bone loss, they show increased TRAP abundance compared to WT animals. These increased TRAP levels in KO vertebrae were restored to WT levels in cohorts treated with $2 \mathrm{mM} \mathrm{K3Citrate}$ and showed a further reduction at $40 \mathrm{mM} \mathrm{K3Citrate} \mathrm{(Figures} \mathrm{7A-E).} \mathrm{Unlike}$ 

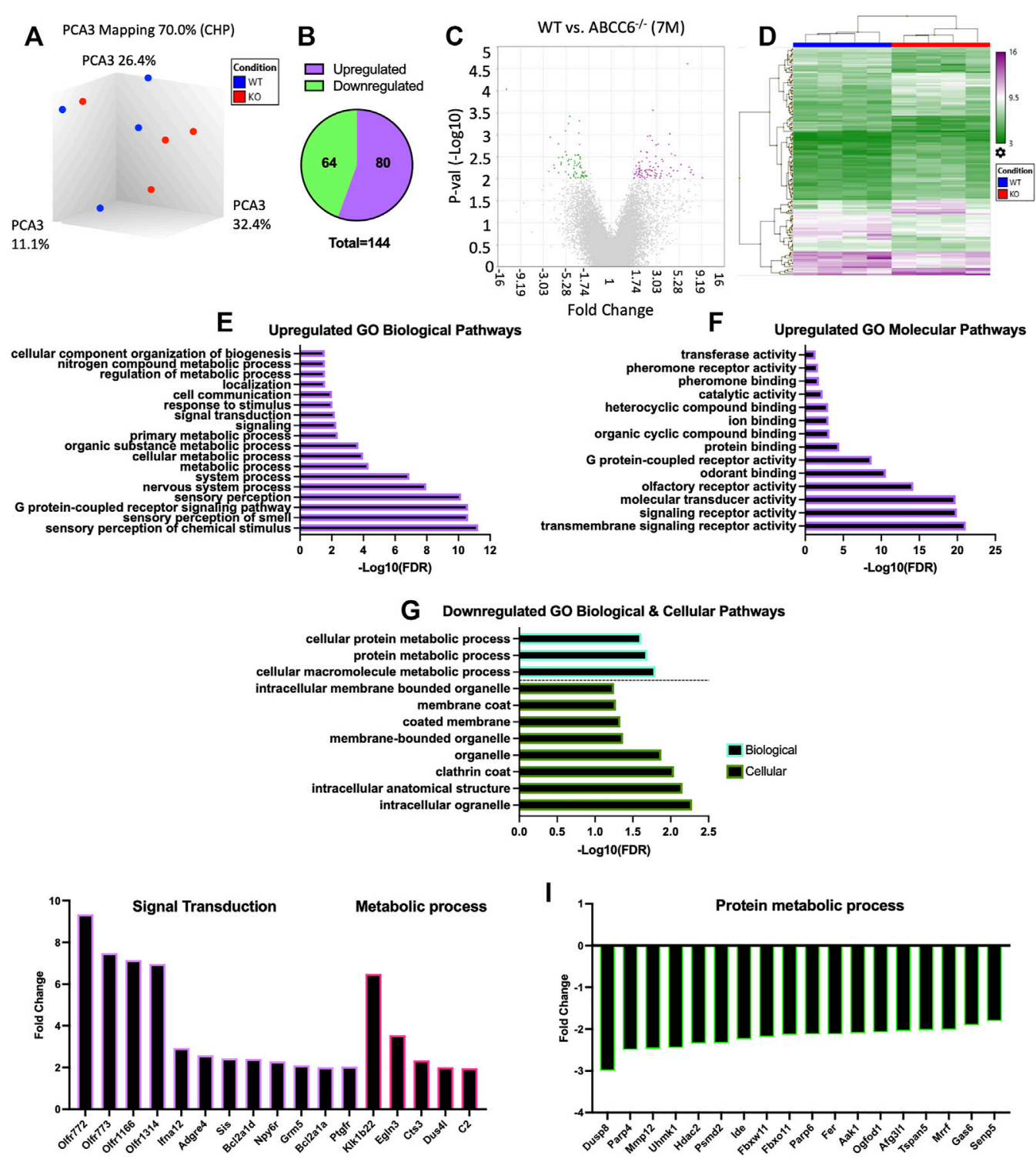

FIGURE 6 | Microarray analysis shows minimal differential expression of genes in $A b c c 6^{-/-}$mice in AF tissue. (A) Principal component analysis showed clustering of samples. (B) Venn diagram showed a total of 144 differentially expressed genes (DEGs) and (C) volcano plot $(p<0.01, \geq 1.75$-fold change). (D) Hierarchical clustering showed the directionality of significant DEGs. Upregulated GO (E) biological processes (BP) and (F) molecular function pathways. (G) Downregulated GO BP and GO cellular component pathways. Representative (I) upregulated and (J) downregulated DEGs from select GO processes.

altered TRAP labeling, at this age, vertebral TNAP activity in $A b c c 6^{-1-}$ was comparable to WT. However, vertebral TNAP abundance was significantly increased in $A b c c \sigma^{/-}$mice treated with $40 \mathrm{mM}$ K3Citrate (Figures 7F-J) suggesting stimulation of osteoblastic function. These results suggest that bone loss from ABCC6 deletion was initiated by elevated osteoclast activity which was subsequently followed by osteoblastic dysfunction.

Biomechanical analysis of lumbar vertebrae from 23-week-old mice revealed that vertebrae of $A b c c \sigma^{1-}$ evidence lower ultimate strain (Figure 7P) and toughness (Figure 7R), suggesting that they experience hampered deformation capability and reduced energy absorption before fracture. Importantly, this reduced bone strength in $A b c c \sigma^{-1-}$ vertebrae was restored when mice were supplemented with $40 \mathrm{mM}$ K3Citrate. There were no differences in stiffness (Figure $\mathbf{7 K}$ ) and ultimate load (Figure 7L) between WT and $A b c c \sigma^{-1-}$ mice or the treatment groups. However, although not statistically significant, similar trends were observed for ultimate displacement (Figure $7 \mathbf{M}$ ), ultimate stress (Figure 70), and ultimate energy (Figure 7Q). At the structural level, $A b c c \sigma^{-1-}$ mice treated with $40 \mathrm{mM}$ K3Citrate evidenced a slight increase in trabecular bone parameters BV/TV, Tb.N., and BMD (Supplementary Figures S4A,C,F) and cortical parameters B.Ar. and MMI (Supplementary Figures S4G,H) over baseline values of age-matched WT mice, suggesting that 

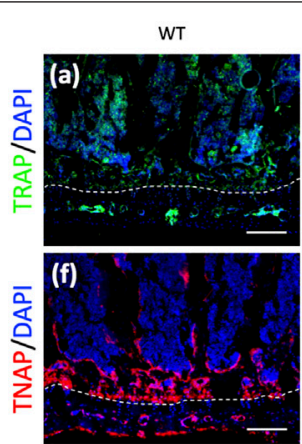

(k)

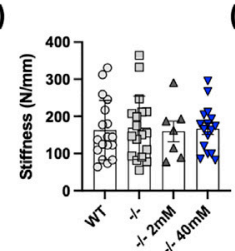

(I)

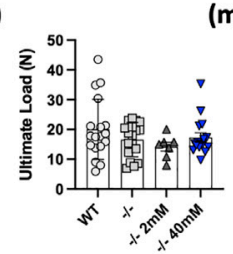

(o)
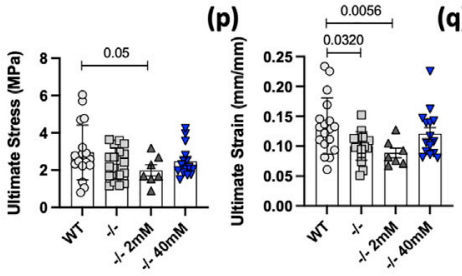

(m)

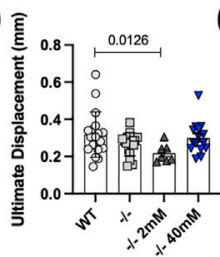

(n)
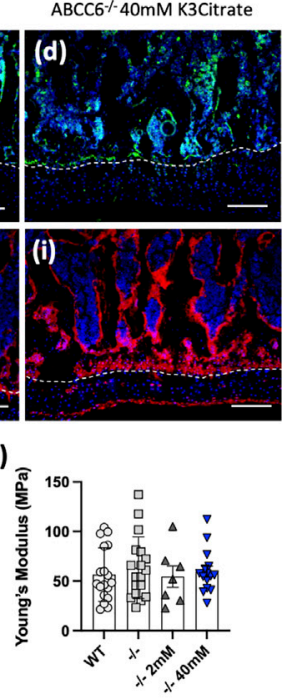

$(r)$
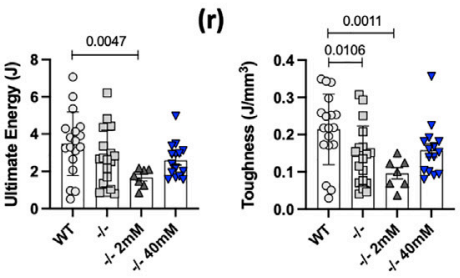

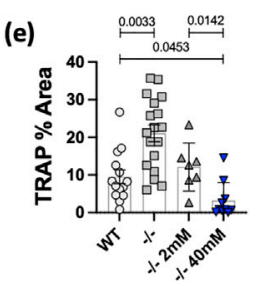

(j)

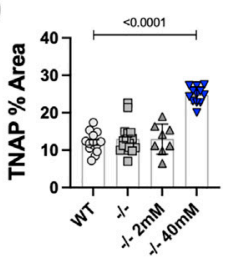

(s)

Abcc6 $\%$ Spinal Phenotype

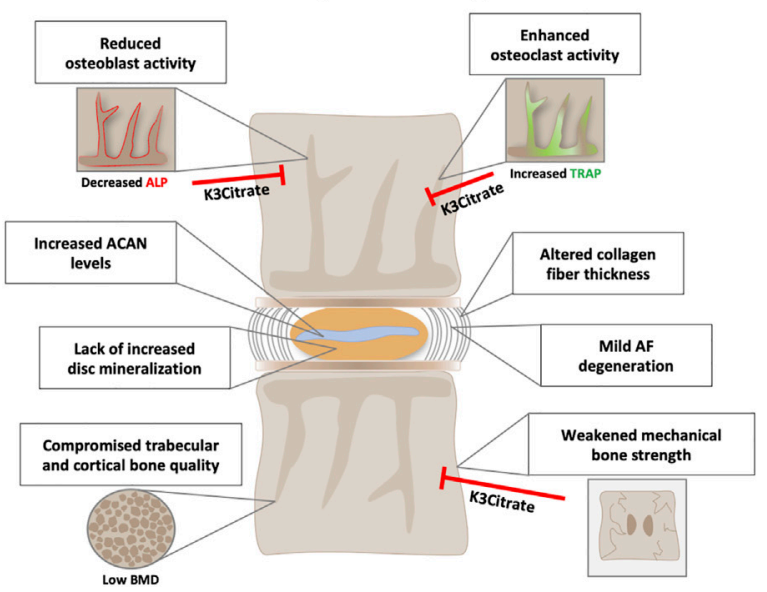

FIGURE 7| Potassium citrate (K3Citrate) treatment blocks osteoclast activation, increases osteoblastic activity, and restores mechanical strength of 23-week-old Abcc6 $6^{-/-}$lumbar vertebrae. IHC staining showed decreased (A-E) TRAP staining and increased (F-J) TNAP staining in Abcc6 $6^{-/-}$lumbar vertebrae after $40 \mathrm{mM}$ K3Citrate treatment. (K-R) Biomechanical analysis revealed a restoration of (P) ultimate strain and (R) toughness in $A_{b c c 6^{-/}}$lumbar vertebrae after $40 \mathrm{mM} \mathrm{K3Citrate}$ treatment. (S) Schematic representing the role of ABCC6 loss on the spinal motion segment and the effects of K3Citrate treatment. Scale bar $=100 \mu \mathrm{m}$. Quantitative analyses are shown as mean $\pm \mathrm{SD}$. ( $n=2$ vertebrae/mice, $n \geq 4$ mice/group). Significance was determined using ANOVA or uncorrected Kruskal-Wallis test as appropriate.

there is some improvement in bone quality as a result of K3Citrate treatment.

Overall, these data suggest that early treatment of K3Citrate improved the cellular and mechanical changes that preceded morphological and structural changes to the bone. Therefore, long-term therapeutic treatment of K3Citrate has the potential benefit of abating trabecular and cortical bone loss by rescuing the mechanical deficit and reverting the cellular changes observed in the vertebrae. Figure $7 \mathrm{~S}$ summarizes the spinal phenotype of $A b c c \sigma^{-1-}$ mice and highlights the potential of $\mathrm{K} 3 \mathrm{Citrate}$ treatment to treat the vertebral defects. 


\section{DISCUSSION}

PXE is an extensively studied mineralization disorder and use of mouse models recapitulating PXE has provided extensive knowledge about ABCC6 function in maintaining the health of elastin-rich tissues that are prone to ectopic mineralization. While dystrophic calcification is one of the known phenotypes of intervertebral disc degeneration, it is unknown whether PXE affects mineralization pathways in the disc and although ABCC6 is not expressed in the spine, its systemic effects on the spine have not been studied in detail. In $A b c c \sigma^{1-}$ mice we found no increase in calcification of the disc, but unexpectedly, these animals had reduced vertebral bone quality and could become a valuable model for gaining further insight into intervertebral disc degeneration (Peng et al., 2021).

There are a limited number of previous studies of patients and mouse models of PXE that have investigated long bone phenotype. A recent clinical study reported that PXE patients have a higher OA score in knee and articular joints (Gielis et al., 2020), however, no effects on long bone mineral density in PXE patients and $A b c c 6$ knockout mice has been noted (Li et al., 2009; Jiang and Uitto, 2012; Martin et al., 2017). This study for the first time observed an osteopenic phenotype in the axial skeleton through a significant reduction of vertebral bone morphology parameters, including reduced trabecular bone volume, thickness, and number as well as reduced bone mineral density. These findings are in line with the early onset osteoporosis and spinal fractures seen in adult patients with heterozygous loss-offunction mutations in ENPP1 and osteoporosis in ENPP1 $1^{-/}$ mice, a model for GACI (Oheim et al., 2020). Likewise, reduced $\mathrm{BMD}$ in the lumbar spine has been noted in $\mathrm{ENT}^{-1-}$ mice, a model for diffuse idiopathic skeletal hyperostosis (DISH) (Hinton et al., 2014; Oheim et al., 2020). These findings suggest that the axial skeleton is sensitive to the impairment of PPi levels. Furthermore, changes in bone turnover markers, such as increased TRAP and reduced TNAP abundance, have been associated with disc degeneration and osteoporosis and are considered valuable tools for understanding the cellular basis of bone loss (Nizet et al., 2020; Xiao et al., 2020). The elevated TRAP abundance observed before the structural deficit in vertebral bone was evident, suggesting that enhanced osteoclastogenesis in $A b c c \sigma^{-1-}$ bone is the driving mechanism for such bone loss and that osteoblastic dysregulation evident by reduced TNAP staining follows osteoclastic activation.

Histological analyses at various ages provided evidence of mild but age-progressive disc degeneration in the AF compartment of $A b c c 6^{-1-}$ mice. Aging promotes degeneration in both the NP and AF compartments (Novais et al., 2020), however only the AF of 16-18-months $\mathrm{Abcc \sigma}^{-}$exhibited a higher incidence of degeneration. While decreased abundance of phenotypic markers CA3 and KRT19 is consistent with aging and degeneration (Lv et al., 2014; Silagi et al., 2018), yet the minor, or lack of, progressive changes observed in $\mathrm{Abcc \sigma}^{\text {l- }}$ mice beyond 7-8 months suggested overall preservation of NP function. Importantly, the lack of major morphological changes in the NP compartment in $A b c c 6^{-1-}$ mice explains the absence of severe deterioration in the overall architecture of the discs. While changes in the $A b c c 6^{-1-}$ disc were relatively mild, the $\mathrm{AF}$ compartment appeared to be more sensitive to the absence of ABCC6, possibly because of the systemic reduction in extracellular PPi levels.

Despite the mild phenotypic differences, transcriptomic analysis showed enrichment of GO processes related to altered signaling and metabolic processes. Of these GO processes, the genes Npy6r, Ptgfr, and Egln3 were upregulated. Npy6r expression is correlated with disc degeneration and has shown to increase expression in AF cells when exposed to inflammatory stimuli (Ashton et al., 1994; Dombrowski et al., 2020). While Ptgfr blocks the interaction of prostaglandin F2-alpha (PGF2 $\alpha$ ) to its receptor, Egln3, or PHD3, modulates NF-кB and HIF-1a signaling pathways and is controlled by both HIF- $1 \alpha$ and proinflammatory cytokines (Fujita et al., 2012; Schoepflin et al., 2016; Silagi et al., 2021). Likewise, expression of MMP12, a promoter of ECM degradation and disc fibrosis (Gruber et al., 2014; Lv et al., 2016), Hdac2 and Tspan5, modulators of RANKL-induced osteoclastogenesis (Iwai et al., 2007; Dou et al., 2016) were all downregulated, suggesting changes in inflammation response genes in the $\mathrm{AF}$ of $A b c c \sigma^{1-}$ discs. However, it is important to note that the relatively small transcriptomic changes in the AF compartment of $A b c c 6^{-1-}$ mice did not contribute to altering biological processes or molecular pathways associated with mineralization and were in line with overall milder phenotypic changes.

Studies of human disc specimens and mouse models have shown a strong correlation among disc calcification, aging, and degeneration status (Warraich et al., 2013; Alvarez-Garcia et al., 2017; Hawellek et al., 2017; Novais et al., 2020), suggesting that dystrophic calcification is one of important sub phenotypes of the degenerative process. Abcc6 null mice experience mineralization in elastin- and/or collagen-rich connective tissues of skin, eyes, and arteries (Bergen et al., 2000) and ENPP1 null mice show hypermineralization of collagen-rich tendons and ligaments (Zhang et al., 2016), suggesting that the elastin and collagenrich AF tissue of the disc could be a potential site of ectopic mineralization in $\mathrm{Abcc6}^{-/-}$mice. A notable observation in our study was that there was no drastic increase in incidence of calcification within the NP and AF compartments of aged $A b c c \sigma^{-1}$ mice. These results suggest that in the avascular environment of the disc, calcium and PPi levels are tightly regulated regardless of the changes in systemic levels of PPi. Relevant to this phenotype, maintenance of extracellular matrix components that may contribute to modulation of mineralization is critical for disc function. For example, COMP, a marker for healthy and young discs is prominently localized in the AF compartment (Molinos et al., 2015) and shown to be an inhibitor of vascular calcification (Du et al., 2011), and the proteoglycan aggrecan inhibits apatite growth through $\mathrm{Ca}^{2+}$ binding (Eanes et al., 1992). Consequently, comparable COMP and ACAN levels in the AF accompanied by a decrease in ACAN turnover and elevated ACAN levels in NP, may suffice to prevent disc mineralization in $A b c c \sigma^{1-}$ mice. It is also important to note that COLX, a protein highly expressed by hypertrophic chondrocytes (Schmid et al., 1990; Roberts et al., 1998), is also a regulator of mineralization in the growth plate through its 
inhibitory actions and is elevated in $A b c c \sigma^{-1-}$ mice (Kirsch et al., 2000).

Considering the importance of balancing the $\mathrm{Pi} / \mathrm{PPi}$ ratio in regulating connective tissue mineralization, it would be expected that a decrement in systemic levels of PPi will result in ectopic mineralization. Ectopic mineralization, however, might be governed by the local vs. systemic regulation of PPi in a tissue-dependent fashion. The tissues usually affected in PXE patients are more reliant on, and therefore more susceptible to, systemic plasma concentrations of PPi. This point is underscored by a recent study comparing the phenotypes of patients with ENPP1 and ABCC6 deficiencies (Ferreira et al., 2021). The authors reported a significantly lower incidence of joint calcification in ABCC6-deficient patients than patients with ENPP1-deficit, suggesting that local regulation of PPi and AMP levels may be critical in ectopic mineralization of cartilaginous tissues (Ferreira et al., 2021). The intervertebral disc, like articular cartilage, is avascular and utilizes the microvasculature of the cartilaginous endplates (CEPs) for metabolite diffusion and likely relies upon locally generated $\mathrm{PPi}$. Previous studies have shown an association between aortic calcification and intervertebral disc height loss in humans (Suri et al., 2012). Disc degeneration also results from nicotine-induced vascular hypertrophy, suggesting that vascular function is important for disc health (Iwahashi et al., 1976). Although we did not investigate the mineralization status of CEP vasculature, based on the CEP morphology and the mild disc phenotype observed it can be presumed that endplate vascular calcification is not a pathology in this model. Notably, progressive ankylosis protein $(\mathrm{ANKH})$, another critical transmembrane protein responsible for regulating mineralization, is highly expressed in osteoblasts, skeletal muscle, as well as disc cells (Ho et al., 2000; Chen et al., 2009; Skybutyte et al., 2010). $\mathrm{ANK}^{\text {ank/ank }}$ mice exhibit a $30 \%$ reduction in plasma $\mathrm{PPi}$, a $65 \%$ reduction in plasma citrate levels and altered long bone geometry along with progressive joint and disc calcification (Sampson and Davis 1976; Sampson 1988; Sampson et al., 1991; Szeri et al., 2020; Szeri et al., 2021). Furthermore, in vitro studies revealed that inhibition of ANKH via TNF increases NP cell mineralization (Krzyzanowska et al., 2021) and ANKH levels are elevated, possibly as a compensatory mechanism in degenerated NP tissues from human patients (Skybutyte et al., 2010), suggesting it may be the predominant player in controlling disc calcification. Therefore, mechanistic investigations of discs from $\mathrm{ANK}^{\text {ank/ank }}$ mice would be important to understand its tissue-specific function and causal effect in disc degeneration and calcification and may provide insights into the key role of locally generated PPi.

Current therapeutic interventions to address ectopic mineralization in disorders such as PXE involve a variety of approaches including PPi supplementation (Dedinszki et al., 2017), bisphosphonates (Kranenburg et al., 2018), statins (Guo et al., 2013), TNAP inhibition (Li et al., 2019), and dietary supplementation of magnesium and citrate (LaRusso et al., 2009; Phillips et al., 2015). Citrate plays an important role in cellular metabolism and about $90 \%$ of the body's source of mobilizable citrate is stored in the bone (Costello et al., 2014), where it chelates calcium and stabilizes apatite nanocrystals for bone formation. In addition to export of citrate via ANK, osteoblasts are responsible for the uptake and storage of citrate, where uptake of plasma citrate relies on sodiumdependent plasma membrane citrate transporter SLC13A5 (Irizarry et al., 2017). Furthermore, reduced bone and plasma citrate levels have been associated with osteoporosis and positively correlate with lumbar BMD (Chen et al., 2018). $A b c c \sigma^{\prime-}$ mice treated with K3Citrate exhibited a dosedependent decrease in TRAP levels, where a low dose normalized TRAP levels to that seen in wild-type vertebrae and a higher dose resulted in further reduction. Although TNAP activity was comparable in 23-week-old WT and untreated $A b c c 6^{-1-}$ mice, TNAP activity significantly increased after $40 \mathrm{mM} \mathrm{K3Citrate}$ treatment. Taken together, these data agree with previous findings where cultures of osteoclasts have shown a dose-dependent reduction of osteoclastogenesis after K3Citrate treatment (Granchi et al., 2017) and suggests that citrate plays a role in regulating both osteoclast differentiation and possibly osteoblast maturation/function. Furthermore, compression analysis of $A b c c \sigma^{1-}$ vertebrae revealed compromised structural and material properties, seen via reduced ultimate strain and toughness showing that the vertebrae are more prone to fracture. After $40 \mathrm{mM}$ K3Citrate treatment, however, there was a restoration of these parameters. This suggests that long-term treatment of K3Citrate is safe and would provide a positive outcome in rescuing vertebral bone quality by promoting direct incorporation of citrate in bone matrix and/or modulating function of bone cells. Moreover, this treatment modality could benefit not only PXE-related changes in vertebrae, but also for other conditions that afflict the spine. In summary, this study provides novel insights into the role of ABCC6 and contribution of systemic PPi in maintaining the health of the intervertebral disc and axial skeleton.

\section{DATA AVAILABILITY STATEMENT}

The datasets presented in this study can be found in online repositories. The names of the repository/repositories and accession number(s) can be found in the article/ Supplementary Material.

\section{ETHICS STATEMENT}

The animal study was reviewed and approved by the Institutional Animal Care and Use Committee.

\section{AUTHOR CONTRIBUTIONS}

$\mathrm{MR}, \mathrm{KV}$, and IS contributed to conceptualization, design, and funding of the study. $\mathrm{PB}, \mathrm{VM}$, and $\mathrm{KV}$ contributed to experimental investigation. $\mathrm{KV}, \mathrm{MR}$, and $\mathrm{RT}$ contributed to the study's methodology. RT, KV, and MR provided the resources for this study. PB and MR wrote the first draft of 
the manuscript. All authors contributed to manuscript revision, read, and approved the submitted version.

\section{FUNDING}

This study is supported by the grants from the National Institute of Arthritis and Musculoskeletal and Skin Disease (NIAMS) R01AR064733, R01AR055655, R01AR074813, and R01AG073349

\section{REFERENCES}

Alvarez-Garcia, O., Matsuzaki, T., Olmer, M., Masuda, K., and Lotz, M. K. (2017). Age-related Reduction in the Expression of FOXO Transcription Factors and Correlations with Intervertebral Disc Degeneration. J. Orthop. Res. 35 (12), 2682-2691. doi:10.1002/jor.23583

Ashton, I. K., Roberts, S., Jaffray, D. C., Polak, J. M., and Eisenstein, S. M. (1994). Neuropeptides in the Human Intervertebral Disc. J. Orthop. Res. 12 (2), 186-192. doi:10.1002/jor.1100120206

Bergen, A. A. B., Plomp, A. S., Schuurman, E. J., Terry, S., Breuning, M., Dauwerse, H., et al. (2000). Mutations in ABCC6 Cause Pseudoxanthoma Elasticum. Nat. Genet. 25 (2), 228-231. doi:10.1038/76109

Borst, P., Váradi, A., and van de Wetering, K. (2019). PXE, a Mysterious Inborn Error Clarified. Trends Biochem. Sci. 44 (2), 125-140. doi:10.1016/j.tibs.2018. 10.005

Chen, H., Wang, Y., Dai, H., Tian, X., Cui, Z.-K., Chen, Z., et al. (2018). Bone and Plasma Citrate Is Reduced in Osteoporosis. Bone 114, 189-197. doi:10.1016/j. bone.2018.06.014

Chen, I.-P., Wang, C. J., Strecker, S., Koczon-Jaremko, B., Boskey, A., and Reichenberger, E. J. (2009). Introduction of a Phe377del mutation in ANK creates a mouse model for craniometaphyseal dysplasia. J. Bone Mineral Res. 24 (7), 1206-1215. doi:10.1359/jbmr.090218

Clarke, M. C. H., Littlewood, T. D., Figg, N., Maguire, J. J., Davenport, A. P., Goddard, M., et al. (2008). Chronic Apoptosis of Vascular Smooth Muscle Cells Accelerates Atherosclerosis and Promotes Calcification and Medial Degeneration. Circ. Res. 102 (12), 1529-1538. doi:10.1161/circresaha.108. 175976

Cloyd, J. M., and Elliott, D. M. (1976). Elastin Content Correlates with Human Disc Degeneration in the Anulus Fibrosus and Nucleus Pulposus. Spine (Phila $\mathrm{Pa}$ 1976) 32 (17), 1826-1831. doi:10.1097/BRS. ob013e3181132a9d

Costello, L. C., Chellaiah, M., Zou, J., Franklin, R. B., and Reynolds, M. A. (2014). The Status of Citrate in the Hydroxyapatite/collagen Complex of Bone; and its Role in Bone Formation. J. Regen. Med. Tissue Eng. 3, 4. doi:10.7243/2050-12183-4

Dedinszki, D., Szeri, F., Kozák, E., Pomozi, V., Tőkési, N., Mezei, T. R., et al. (2017). Oral Administration of Pyrophosphate Inhibits Connective Tissue Calcification. EMBO Mol. Med. 9 (11), 1463-1470. doi:10.15252/emmm. 201707532

Dombrowski, M. E., Olsen, A. S., Vaudreuil, N., Couch, B. K., Dong, Q., Tucci, M., et al. (2020). Rabbit Annulus Fibrosus Cells Express Neuropeptide Y, Which Is Influenced by Mechanical and Inflammatory Stress. Neurospine 17 (1), 69-76. doi:10.14245/ns.2040046.023

Dou, C., Li, N., Ding, N., Liu, C., Yang, X., Kang, F., et al. (2016). HDAC2 Regulates FoxO1 during RANKL-Induced Osteoclastogenesis. Am. J. Physiology-Cell Physiol. 310 (10), C780-C787. doi:10.1152/ajpcell.00351.2015

Du, Y., Wang, Y., Wang, L., Liu, B., Tian, Q., Liu, C.-j., et al. (2011). Cartilage Oligomeric Matrix Protein Inhibits Vascular Smooth Muscle Calcification by Interacting with Bone Morphogenetic Protein-2. Circ. Res. 108 (8), 917-928. doi:10.1161/circresaha.110.234328

Dyment, N. A., Jiang, X., Chen, L., Hong, S.-H., Adams, D. J., Ackert-Bicknell, C., et al. (2016). High-Throughput, Multi-Image Cryohistology of Mineralized Tissues. JoVE (115), 54468. doi:10.3791/54468 to MR. KV receives funding from PXE International and the NIAMS, R01AR072695.

\section{SUPPLEMENTARY MATERIAL}

The Supplementary Material for this article can be found online at: https://www.frontiersin.org/articles/10.3389/fcell.2022.823249/ full\#supplementary-material

Eanes, E. D., Hailer, A. W., Midura, R. J., and Hascall, V. C. (1992). Proteoglycan Inhibition of Calcium Phosphate Precipitation in Liposomal Suspensions. Glycobiology 2 (6), 571-578. doi:10.1093/glycob/2.6.571

Favre, G., Laurain, A., Aranyi, T., Szeri, F., Fulop, K., Le Saux, O., et al. (2017). The ABCC6 Transporter: A New Player in Biomineralization. Int. J. Mol. Sci. 18 (9), 1941. doi:10.3390/ijms18091941

Ferreira, C. R., Kintzinger, K., Hackbarth, M. E., Botschen, U., Nitschke, Y., Mughal, M. Z., et al. (2021). Ectopic Calcification and Hypophosphatemic Rickets: Natural History of ENPP1 and ABCC6 Deficiencies. J. Bone Miner Res. 36 (11), 2193-2202. doi:10.1002/jbmr.4418

Fournier, D. E., Kiser, P. K., Beach, R. J., Dixon, S. J., and Séguin, C. A. (2020). Dystrophic Calcification and Heterotopic Ossification in Fibrocartilaginous Tissues of the Spine in Diffuse Idiopathic Skeletal Hyperostosis (DISH). Bone Res. 8 (1), 16. doi:10.1038/s41413-020-0091-6

Fujita, N., Gogate, S. S., Chiba, K., Toyama, Y., Shapiro, I. M., and Risbud, M. V. (2012). Prolyl Hydroxylase 3 (PHD3) Modulates Catabolic Effects of Tumor Necrosis Factor- $\alpha$ (TNF- $\alpha$ ) on Cells of the Nucleus Pulposus through Co-

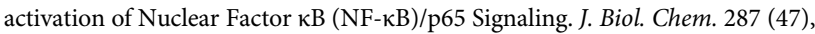
39942-39953. doi:10.1074/jbc.m112.375964

Gielis, W. P., de Jong, P. A., Bartstra, J. W., Foppen, W., Spiering, W., and den Harder, A. M. (2020). Osteoarthritis in Pseudoxanthoma Elasticum Patients: An Explorative Imaging Study. Jcm 9 (12), 3898. doi:10.3390/jcm9123898

Granchi, D., Torreggiani, E., Massa, A., Caudarella, R., Di Pompo, G., and Baldini, N. (2017). Potassium Citrate Prevents Increased Osteoclastogenesis Resulting from Acidic Conditions: Implication for the Treatment of Postmenopausal Bone Loss. PLoS One 12 (7), e0181230. doi:10.1371/journal.pone.0181230

Gruber, H. E., Ingram, J. A., Cox, M. D., and Hanley, E. N., Jr (2014). Matrix Metalloproteinase-12 Immunolocalization in the Degenerating Human Intervertebral Disc and Sand Rat Spine: Biologic Implications. Exp. Mol. Pathol. 97 (1), 1-5. doi:10.1016/j.yexmp.2014.04.007

Guo, H., Li, Q., Chou, D. W., and Uitto, J. (2013). Atorvastatin Counteracts Aberrant Soft Tissue Mineralization in a Mouse Model of Pseudoxanthoma Elasticum (Abcc6 -/- ). J. Mol. Med. 91 (10), 1177-1184. doi:10.1007/s00109013-1066-5

Hawellek, T., Hubert, J., Hischke, S., Rolvien, T., Krause, M., Püschel, K., et al. (2017). Microcalcification of Lumbar Spine Intervertebral Discs and Facet Joints Is Associated with Cartilage Degeneration, but Differs in Prevalence and its Relation to Age. J. Orthop. Res. 35 (12), 2692-2699. doi:10.1002/jor. 23591

Hinton, D. J., McGee-Lawrence, M. E., Lee, M. R., Kwong, H. K., Westendorf, J. J., and Choi, D.-S. (2014). Aberrant Bone Density in Aging Mice Lacking the Adenosine Transporter ENT1. PLoS One 9 (2), e88818. doi:10.1371/journal. pone. 0088818

Ho, A. M., Johnson, M. D., and Kingsley, D. M. (2000). Role of the Mouse Ank Gene in Control of Tissue Calcification and Arthritis. Science 289 (5477), 265-270. doi:10.1126/science.289.5477.265

Hristova, G. I., Jarzem, P., Ouellet, J. A., Roughley, P. J., Epure, L. M., Antoniou, J., et al. (2011). Calcification in Human Intervertebral Disc Degeneration and Scoliosis. J. Orthop. Res. 29 (12), 1888-1895. doi:10.1002/jor.21456

Hu, Y. Y., Rawal, A., and Schmidt-Rohr, K. (2010). Strongly Bound Citrate Stabilizes the Apatite Nanocrystals in Bone. Proc. Natl. Acad. Sci. 107 (52), 22425-22429. doi:10.1073/pnas.1009219107

Ii, H., Warraich, S., Tenn, N., Quinonez, D., Holdsworth, D. W., Hammond, J. R., et al. (2016). Disruption of Biomineralization Pathways in Spinal Tissues of a 
Mouse Model of Diffuse Idiopathic Skeletal Hyperostosis. Bone 90, 37-49. doi:10.1016/j.bone.2016.05.008

Irizarry, A. R., Yan, G., Zeng, Q., Lucchesi, J., Hamang, M. J., Ma, Y. L., et al. (2017). Defective Enamel and Bone Development in Sodium-dependent Citrate Transporter (NaCT) Slc13a5 Deficient Mice. PLoS One 12 (4), e0175465. doi:10.1371/journal.pone.0175465

Iwahashi, M., Matsuzaki, H., Tokuhashi, Y., Wakabayashi, K., and Uematsu, Y. (1976). Mechanism of Intervertebral Disc Degeneration Caused by Nicotine in Rabbits to Explicate Intervertebral Disc Disorders Caused by Smoking. Spine (Phila Pa 1976) 27 (13), 1396-1401. doi:10.1097/00007632-20020701000005

Iwai, K., Ishii, M., Ohshima, S., Miyatake, K., and Saeki, Y. (2007). Expression and Function of Transmembrane-4 Superfamily (Tetraspanin) Proteins in Osteoclasts: Reciprocal Roles of Tspan-5 and NET-6 during Osteoclastogenesis. Allergol. Int. 56 (4), 457-463. doi:10.2332/allergolint.o07-488

Jansen, R. S., Duijst, S., Mahakena, S., Sommer, D., Szeri, F., Váradi, A., et al. (2014). ABCC6-mediated ATP Secretion by the Liver Is the Main Source of the Mineralization Inhibitor Inorganic Pyrophosphate in the Systemic Circulation-Brief Report. Atvb 34 (9), 1985-1989. doi:10.1161/atvbaha.114. 304017

Jansen, R. S., Kucukosmanoglu, A., de Haas, M., Sapthu, S., Otero, J. A., Hegman, I. E. M., et al. (2013). ABCC6 Prevents Ectopic Mineralization Seen in Pseudoxanthoma Elasticum by Inducing Cellular Nucleotide Release. Proc. Natl. Acad. Sci. 110 (50), 20206-20211. doi:10.1073/pnas.1319582110

Jiang, Q., and Uitto, J. (2012). Restricting Dietary Magnesium Accelerates Ectopic Connective Tissue Mineralization in a Mouse Model of Pseudoxanthoma elasticum(Abcc6-/-). Exp. Dermatol. 21 (9), 694-699. doi:10.1111/j.16000625.2012.01553.x

Kirsch, T., Harrison, G., Golub, E. E., and Nah, H.-D. (2000). The Roles of Annexins and Types II and X Collagen in Matrix Vesicle-Mediated Mineralization of Growth Plate Cartilage. J. Biol. Chem. 275 (45), 35577-35583. doi:10.1074/jbc.m005648200

Klement, J. F., Matsuzaki, Y., Jiang, Q.-J., Terlizzi, J., Choi, H. Y., Fujimoto, N., et al. (2005). Targeted Ablation of the Abcc6 Gene Results in Ectopic Mineralization of Connective Tissues. Mol. Cel Biol 25 (18), 8299-8310. doi:10.1128/mcb.25. $18.8299-8310.2005$

Kranenburg, G., de Jong, P. A., Bartstra, J. W., Lagerweij, S. J., Lam, M. G., Ossewaarde-van Norel, J., et al. (2018). Etidronate for Prevention of Ectopic Mineralization in Patients with Pseudoxanthoma Elasticum. J. Am. Coll. Cardiol. 71 (10), 1117-1126. doi:10.1016/j.jacc.2017.12.062

Krzyzanowska, A. K., Frawley, R. J., Damle, S., Chen, T., Otero, M., and Cunningham, M. E. (2021). Activation of Nuclear Factor-Kappa B by TNF Promotes Nucleus Pulposus Mineralization through Inhibition of ANKH and ENPP1. Sci. Rep. 11 (1), 8271. doi:10.1038/s41598-021-87665-2

LaRusso, J., Li, Q., Jiang, Q., and Uitto, J. (2009). Elevated Dietary Magnesium Prevents Connective Tissue Mineralization in a Mouse Model of Pseudoxanthoma Elasticum (Abcc6-/-). J. Invest. Dermatol. 129 (6), 1388-1394. doi:10.1038/jid.2008.391

Li, Q., Jiang, Q., and Uitto, J. (2014). Ectopic Mineralization Disorders of the Extracellular Matrix of Connective Tissue: Molecular Genetics and Pathomechanisms of Aberrant Calcification. Matrix Biol. 33, 23-28. doi:10. 1016/j.matbio.2013.06.003

Li, Q., Kingman, J., van de Wetering, K., Tannouri, S., Sundberg, J. P., and Uitto, J. (2017). Abcc6 Knockout Rat Model Highlights the Role of Liver in PPi Homeostasis in Pseudoxanthoma Elasticum. J. Invest. Dermatol. 137 (5), 1025-1032. doi:10.1016/j.jid.2016.11.042

Li, Q., Larusso, J., Grand-Pierre, A. E., and Uitto, J. (2009). Magnesium CarbonateContaining Phosphate Binder Prevents Connective Tissue Mineralization inAbcc6ẩ/ẩ Miceâ€"Potential for Treatment of Pseudoxanthoma Elasticum. Clin. Transl Sci. 2 (6), 398-404. doi:10.1111/j.1752-8062.2009.00161.x

Li, Q., van de Wetering, K., and Uitto, J. (2019). Pseudoxanthoma Elasticum as a Paradigm of Heritable Ectopic Mineralization Disorders. Am. J. Pathol. 189 (2), 216-225. doi:10.1016/j.ajpath.2018.09.014

Lv, F.-J., Peng, Y., Lim, F. L., Sun, Y., Lv, M., Zhou, L., et al. (2016). Matrix Metalloproteinase 12 Is an Indicator of Intervertebral Disc Degeneration Coexpressed with Fibrotic Markers. Osteoarthritis and Cartilage 24 (10), 1826-1836. doi:10.1016/j.joca.2016.05.012
Lv, F., Leung, V. Y. L., Huang, S., Huang, Y., Sun, Y., and Cheung, K. M. C. (2014). In Search of Nucleus Pulposus-specific Molecular Markers. Rheumatology 53 (4), 600-610. doi:10.1093/rheumatology/ket303

Mackay, E. W., Apschner, A., and Schulte-Merker, S. (2015). Vitamin K Reduces Hypermineralisation in Zebrafish Models of PXE and GACI. Development 142 (6), 1095-1101. doi:10.1242/dev.113811

Martin, L., Hoppé, E., Kauffenstein, G., Omarjee, L., Navasiolava, N., Henni, S., et al. (2017). Early Arterial Calcification Does Not Correlate with Bone Loss in Pseudoxanthoma Elasticum. Bone 103, 88-92. doi:10.1016/j.bone.2017.06.017

Molinos, M., Almeida, C. R., Caldeira, J., Cunha, C., Gonçalves, R. M., and Barbosa, M. A. (2015). Inflammation in Intervertebral Disc Degeneration and Regeneration. J. R. Soc. Interf. 12 (104), 20141191. doi:10.1098/rsif.2014.1191

Moskowitz, R. W., Ziv, I., Denko, C. W., Boja, B., Jones, P. K., and Adler, J. H. (1990). Spondylosis in Sand Rats: a Model of Intervertebral Disc Degeneration and Hyperostosis. J. Orthop. Res. 8 (3), 401-411. doi:10.1002/jor.1100080312

Nizet, A., Cavalier, E., Stenvinkel, P., Haarhaus, M., and Magnusson, P. (2020). Bone Alkaline Phosphatase: An Important Biomarker in Chronic Kidney Disease - mineral and Bone Disorder. Clinica Chim. Acta 501, 198-206. doi:10.1016/j.cca.2019.11.012

Novais, E. J., Tran, V. A., Miao, J., Slaver, K., Sinensky, A., Dyment, N. A., et al. (2020). Comparison of Inbred Mouse Strains Shows Diverse Phenotypic Outcomes of Intervertebral Disc Aging. Aging Cell 19 (5), e13148. doi:10. 1111/acel.13148

Oheim, R., Zimmerman, K., Maulding, N. D., Stürznickel, J., von Kroge, S., Kavanagh, D., et al. (2020). Human Heterozygous ENPP1 Deficiency Is Associated with Early Onset Osteoporosis, a Phenotype Recapitulated in a Mouse Model of Enpp1 Deficiency. J. Bone Miner Res. 35 (3), 528-539. doi:10. 1002/jbmr.3911

Park, J., Fertala, A., and Tomlinson, R. E. (2019). Naproxen Impairs Load-Induced Bone Formation, Reduces Bone Toughness, and Diminishes Woven Bone Formation Following Stress Fracture in Mice. Bone 124, 22-32. doi:10.1016/ j.bone.2019.04.009

Peng, Y., Qing, X., Shu, H., Tian, S., Yang, W., and Chen, S. (2021). Proper Animal Experimental Designs for Preclinical Research of Biomaterials for Intervertebral Disc Regeneration. Biomater. Translational 2 (2), 91-142. doi:10.12336/ biomatertransl.2021.02.003

Phillips, R., Hanchanale, V. S., Myatt, A., Somani, B., Nabi, G., and Biyani, C. S. (2015). Citrate Salts for Preventing and Treating Calcium Containing Kidney Stones in Adults. Cochrane Database Syst. Rev. (10), CD010057. doi:10.1002/ 14651858.CD010057.pub2

Roberts, S., Bains, M. A., Kwan, A., Menage, J., and Eisenstein, S. M. (1998). Type X Collagen in the Human Invertebral Disc: an Indication of Repair or Remodelling. Histochem. J. 30 (2), 89-95. doi:10.1023/a:1003278915981

Sampson, H. W., and Davis, J. S. (1976). Histopathology of the Intervertebral Disc of Progressive Ankylosis Mice. Spine (Phila Pa 1976) 13 (6), 650-654.

Sampson, H. W., Davis, R. W., and Dufner, D. C. (1991). Spondyloarthropathy in Progressive Ankylosis Mice: Ultrastructural Features of the Intervertebral Disk. Acta Anatomica 141 (1), 36-41. doi:10.1159/000147096

Sampson, H. W. (1988). Ultrastructure of the Mineralizing Metacarpophalangeal Joint of Progressive Ankylosis (Ank/ank) Mice. Am. J. Anat. 182 (3), 257-269. doi:10.1002/aja.1001820307

Schmid, T. M., Popp, R. G., and Linsenmayer, T. F. (1990). Hypertrophic Cartilage Matrix. Ann. NY Acad. Sci. 580, 64-73. doi:10.1111/j.1749-6632.1990.tb17918.x

Schoepflin, Z. R., Shapiro, I. M., and Risbud, M. V. (2016). Class I and IIa HDACs Mediate HIF-1a Stability through PHD2-dependent Mechanism, while HDAC6, a Class IIb Member, Promotes HIF-1a Transcriptional Activity in Nucleus Pulposus Cells of the Intervertebral Disc. J. Bone Miner Res. 31 (6), 1287-1299. doi:10.1002/jbmr.2787

Silagi, E. S., Batista, P., Shapiro, I. M., and Risbud, M. V. (2018). Expression of Carbonic Anhydrase III, a Nucleus Pulposus Phenotypic Marker, Is HypoxiaResponsive and Confers Protection from Oxidative Stress-Induced Cell Death. Sci. Rep. 8 (1), 4856. doi:10.1038/s41598-018-23196-7

Silagi, E. S., Schipani, E., Shapiro, I. M., and Risbud, M. V. (2021). The Role of HIF Proteins in Maintaining the Metabolic Health of the Intervertebral Disc. Nat. Rev. Rheumatol. 17 (7), 426-439. doi:10.1038/s41584-021-00621-2

Skubutyte, R., Markova, D., Freeman, T. A., Anderson, D. G., Dion, A. S., Williams, C. J., et al. (2010). Hypoxia-inducible Factor Regulation of ANK Expression in Nucleus Pulposus Cells: Possible Implications in Controlling Dystrophic 
Mineralization in the Intervertebral Disc. Arthritis Rheum. 62 (9), 2707-2715. doi:10.1002/art.27558

Suri, P., Hunter, D. J., Rainville, J., Guermazi, A., and Katz, J. N. (2012). Quantitative Assessment of Abdominal Aortic Calcification and Associations with Lumbar Intervertebral Disc Height Loss: the Framingham Study. Spine J. 12 (4), 315-323. doi:10.1016/j.spinee.2012.03.033

Szeri, F., Niaziorimi, F., Donnelly, S., Fariha, N., Tertshnaia, M., Patel, D., et al. (2021). Ankylosis Homologue Mediates Cellular Efflux of ATP, Not Pyrophosphate. bioRxiv. doi:10.1101/2021.08.27.457978

Szeri, F., Lundkvist, S., Donnelly, S., Engelke, U. F. H., Rhee, K., Williams, C. J., et al. (2020). The Membrane Protein ANKH Is Crucial for Bone Mechanical Performance by Mediating Cellular export of Citrate and ATP. Plos Genet. 16 (7), e1008884. doi:10.1371/journal.pgen.1008884

Tessier, S., Tran, V. A., Ottone, O. K., Novais, E. J., Doolittle, A., DiMuzio, M. J., et al. (2020). TonEBP-deficiency Accelerates Intervertebral Disc Degeneration Underscored by Matrix Remodeling, Cytoskeletal Rearrangements, and Changes in Proinflammatory Gene Expression. Matrix Biol. 87, 94-111. doi:10.1016/j.matbio.2019.10.007

Tsingas, M., Ottone, O. K., Haseeb, A., Barve, R. A., Shapiro, I. M., Lefebvre, V., et al. (2020). Sox9 Deletion Causes Severe Intervertebral Disc Degeneration Characterized by Apoptosis, Matrix Remodeling, and Compartment-specific Transcriptomic Changes. Matrix Biol. 94, 110-133. doi:10.1016/j.matbio.2020.09.003

Urban, J. P., Smith, S., and Fairbank, J. C. (1976). Nutrition of the Intervertebral Disc. Spine (Phila Pa 1976) 29 (23), 2700-2709. doi:10.1097/01.brs.0000146499. 97948.52

Warraich, S., Bone, D. B., Quinonez, D., Ii, H., Choi, D.-S., Holdsworth, D. W., et al. (2013). Loss of Equilibrative Nucleoside Transporter 1 in Mice Leads to Progressive Ectopic Mineralization of Spinal Tissues Resembling Diffuse Idiopathic Skeletal Hyperostosis in Humans. J. Bone Miner Res. 28 (5), 1135-1149. doi:10.1002/jbmr.1826
Xiao, Z.-f., Su, G.-y., Hou, Y., Chen, S.-d., Zhao, B.-d., He, J.-b., et al. (2020). Mechanics and Biology Interact in Intervertebral Disc Degeneration: A Novel Composite Mouse Model. Calcif Tissue Int. 106 (4), 401-414. doi:10.1007/ s00223-019-00644-8

Yu, J., Tirlapur, U., Fairbank, J., Handford, P., Roberts, S., Winlove, C. P., et al. (2007). Microfibrils, Elastin Fibres and Collagen Fibres in the Human Intervertebral Disc and Bovine Tail Disc. J. Anat. 210 (4), 460-471. doi:10. 1111/j.1469-7580.2007.00707.x

Zhang, J., Dyment, N. A., Rowe, D. W., Siu, S. Y., Sundberg, J. P., Uitto, J., et al. (2016). Ectopic Mineralization of Cartilage and Collagen-Rich Tendons and Ligaments in Enpplasj-2J Mice. Oncotarget 7 (11), 12000-12009. doi:10.18632/ oncotarget. 7455

Conflict of Interest: The authors declare that the research was conducted in the absence of any commercial or financial relationships that could be construed as a potential conflict of interest.

Publisher's Note: All claims expressed in this article are solely those of the authors and do not necessarily represent those of their affiliated organizations, or those of the publisher, the editors and the reviewers. Any product that may be evaluated in this article, or claim that may be made by its manufacturer, is not guaranteed or endorsed by the publisher.

Copyright (๔) 2022 Boneski, Madhu, Tomlinson, Shapiro, van de Wetering and Risbud. This is an open-access article distributed under the terms of the Creative Commons Attribution License (CC BY). The use, distribution or reproduction in other forums is permitted, provided the original author(s) and the copyright owner(s) are credited and that the original publication in this journal is cited, in accordance with accepted academic practice. No use, distribution or reproduction is permitted which does not comply with these terms. 